\title{
Chronic use of teething gel causing salicylate toxicity
}

\author{
Authors: \\ Dr Trung Nguyen ${ }^{1,4}$ \\ Assoc Prof Noel Cranswick ${ }^{1,2}$ \\ Dr Jeremy Rosenbaum ${ }^{3}$ \\ Dr Ben Gelbart ${ }^{4}$ \\ Dr Shidan Tosif ${ }^{1}$
}

\begin{abstract}
${ }^{1}$ Departments of General Medicine, ${ }^{2}$ Clinical Pharmacology and ${ }^{3}$ Gastroenterology, and ${ }^{4}$ Paediatric Intensive Care Unit, The Royal Children's Hospital Melbourne, 50 Flemington Road, Parkville, Victoria, Australia 3052
\end{abstract}

Correspondence: Dr Trung Nguyen, Department of General Medicine, Royal Children's Hospital, 3 West Clinical Offices, 50 Flemington Road, Parkville, Vic. 3052, Australia. Fax: +61 39345 4751; email: trung.nguyen@rch.org.au

\section{Key points}

- Teething gels are commonly used for symptoms of tooth eruption despite lack of evidence of benefit and potential harm from salicylate poisoning.

- Salicylate poisoning can occur from systemic absorption with frequent use of teething gels containing choline salicylate.

- Enquiring about medication usage is essential when taking a history, and rapid recognition of salicylate poisoning is essential in order to initiate prompt life-saving supportive treatment.

- Greater warnings are required for awareness of potential drug toxicity from teething gels.

This is the author manuscript accepted for publication and has undergone full peer review but has not been through the copyediting, typesetting, pagination and proofreading process, which may lead to differences between this version and the Version of Record. Please cite this article as doi: $10.1111 /$ jpc.13861

This article is protected by copyright. All rights reserved. 
Teething gels are commonly used for symptoms attributed to primary tooth eruption in infancy despite lack of benefit and potential harm from chronic salicylate poisoning. We describe a case of metabolic derangement with significant encephalopathy and liver impairment secondary to chronic use of Bonjela teething gel (Reckitt Benckiser [8.7\% choline salicylate]) in a 17 month old boy. We summarise relevant pharmacological considerations, and highlight the need for greater warning on packaging of teething gels for awareness of this risk. This case acts as a useful clinical vignette with which to highlight the importance of taking a detailed medication history, including use of over-thecounter medications, and of considering adverse effects of medications as a cause of presentation in the initial assessment of a sick child with metabolic derangement of unclear aetiology.

\section{Case Report}

A previously well 17-month-old boy presented to the Emergency Department of a regional hospital after being found unresponsive, following a forty-eight hour illness consisting of cough, coryza, vomiting and diarrhoea but no documented fever. He had received ibuprofen $100 \mathrm{mg}$ every 6 hours $(9.3 \mathrm{mg} / \mathrm{kg} / \mathrm{dose})$ for 24 hours prior to presentation. The family denied administration of any other medications.

On arrival, he had generalised hypotonia and was responsive only to painful stimulus. His pupils were dilated at $5 \mathrm{~mm}$ and reactive to light bilaterally. Vital signs included a heart rate of 114 beats per minute, blood pressure of $90 / 50 \mathrm{mmHg}$, and he had tachypnoea and hyperpnoea with a respiratory rate of 60 breaths per minute. He did not have a fever. Auscultation of heart and lungs was normal. Abdominal palpation revealed no organomegaly, and there was no jaundice, rash or bruising. Initial therapies included intubation and mechanical ventilation for decreased conscious state, fluid resuscitation and inotropic support, and broad-spectrum antibiotics and antivirals for potential infectious aetiology. Investigations revealed a compensated lactic acidosis, transaminitis, hyperammonaemia and hypoglycaemia. The biochemical results are outlined in Table 1. Computedtomography of the brain was unremarkable.

He was transferred to the Paediatric Intensive Care Unit (PICU) of our institution. Veno-venous haemofiltration was initiated for management of hyperammonaemia and acute liver failure.

Supportive therapies included fresh frozen plasma and vitamin $\mathrm{K}$ for correction of coagulopathy, and dextrose infusions for hypoglycaemia. 
A urine drug screen was performed which demonstrated salicylate metabolites. Serum salicylate level was supratherapeutic at $470 \mathrm{mg} / \mathrm{L}$ (normal $<300 \mathrm{mg} / \mathrm{L}$ ) and urine alkalinisation was commenced. Nasopharyngeal swab for viral polymerase chain reaction demonstrated parainfluenza. Magnetic resonance imaging of the brain was unremarkable. Cultures of blood, urine and cerebrospinal fluid were negative, and no leukocytes were detected in the cerebrospinal fluid. Liver ultrasound demonstrated a normal-size liver with increased echogenicity. Investigations for an inborn error of metabolism were negative.

The family denied having administered aspirin, but further history revealed the use of Bonjela teething gel (Reckitt Benckiser [8.7\% choline salicylate]) for seven months. The patient's mother stated she would place several drops onto her fingers and rub it into his gums, once or twice daily. She estimated the use of one tube every one to two weeks.

With supportive therapies, his salicylate level improved and was $<30 \mathrm{mg} / \mathrm{L}$ at 48 hours. Transaminitis, hyperammonaemia and coagulopathy also improved after 48 hours and his conscious state returned to normal. He was extubated on day 2 of admission, discharged from PICU on day 4 and from hospital on day 7. Serum alanine aminotransferase level had improved to $120 \mathrm{IU} / \mathrm{L}$ at time of discharge. Follow up at six months suggested complete recovery.

\section{Discussion}

We describe the case of chronic salicylate poisoning from the use of teething gel manifesting as severe encephalopathy and liver impairment, with lactic acidosis, hyperammonaemia and coagulopathy, ${ }^{1}$ in the setting of a parainfluenza infection. The features of this case are consistent with a Reye-like syndrome.

First described in 1963, Reye syndrome is a rare clinical entity that manifests as a combination of liver disease and non-inflammatory encephalopathy. ${ }^{2,3}$ A prodromal viral illness is often described in Reye syndrome, with influenza and varicella viruses being most common, although parainfluenza virus has also been implicated. ${ }^{4}$ Aspirin (acetylsalicylic acid) usage during the prodromal illness has long been linked to development of Reye syndrome, leading to recommendations to avoid use of aspirin in children for viral illnesses. More recently, a case report suggested choline salicylate (a non- 
acetyl salicylate compound) is also implicated. ${ }^{5}$ However, in that particular case report, the patient described had normal ammonia levels which would be more consistent with salicylate toxicity rather than Reye syndrome, as plasma ammonia concentration increased greater than 3-times upper limit of normal is a necessary criteria for clinical diagnosis of Reye syndrome. ${ }^{3,6}$ Although features of our case would appear to meet the Centers for Disease Control (CDC) clinical criteria for Reye syndrome (see Table 2), the CDC criteria for Reye syndrome is fairly non-specific, and other conditions can account for the cerebral and hepatic abnormalities outlined in the CDC criteria. Reye-like syndromes include drug toxicity, infections, infection-related conditions, and some inborn errors of metabolism.

Although salicylate toxicity is uncommonly encountered, it should be considered as a potential diagnosis in a child presenting with encephalopathy and liver impairment, especially if exposure to salicylate-containing medication is suspected, highlighting the importance of enquiring about medications when taking a history. As described, inborn errors of metabolism, particularly fatty acid oxidation disorders, can manifest similarly to salicylate toxicity in their presentation and need to be excluded. ${ }^{7}$ Causes of hepatic encephalopathy also need to be considered as differential diagnoses in a child presenting with hyperammonaemia and liver impairment. Prompt recognition of salicylate intoxication and aggressive management in the intensive care unit is vital as clinical status can deteriorate rapidly. Urine alkalinisation enhances the ionisation of acidic drugs like salicylates and therefore promotes renal excretion, and correction of hyperammonaemia with haemofiltration is essential. ${ }^{1}$

Symptoms that are frequently attributed to primary tooth eruption in toddlers (for example, drooling, sleep disturbance, irritability, ear tugging, reduced oral intake, and fever) are often the cause of much distress for parents, prompting use of therapies such as teething gels despite little evidence of benefit. ${ }^{8}$ Choline salicylate in teething gels shares anti-inflammatory properties with aspirin. A tube of Bonjela $(15 \mathrm{~g})$ contains $1.31 \mathrm{~g}$ of choline salicylate which is equivalent to approximately $930 \mathrm{mg}$ of aspirin. ${ }^{6}$ Product information states: 'Apply a small amount of Bonjela Teething Gel with a clean finger to the affected area and rub in gently ... application can be repeated every 3 hours as necessary'. ${ }^{9}$ This dosing method is imprecise, difficult to monitor and can equate to the use of one tube every three days. ${ }^{10}$ Moreover, it has been suggested that the symptomatic relief attributed to the use of teething gels is due to the direct pressure of application to irritated areas of the mucous membrane rather than the anti-inflammatory effects of choline salicylate. ${ }^{8}$ 
Our case of salicylate toxicity secondary to chronic use of choline salicylate-containing teething gel presenting with features of a Reye-like syndrome is not the first such case described. ${ }^{11}$ Topical salicylate-based teething gels are contraindicated in those under the age of 16 in the United Kingdom (UK) following concerns of chronic toxicity. ${ }^{6}$ As a result, Bonjela teething gel in the UK contains lignocaine as its analgesic component instead of choline salicylate. Greater awareness of the potential for salicylate toxicity is required. We recommend warnings on product information and consideration of removing salicylate from teething gels in order to avoid inadvertent life-threatening drug toxicity.

\section{Multiple choice questions}

1. Which of the following approaches for management of teething is NOT recommended?
a. Teething rings
b. Rubbing the infant's gums with a clean finger
c. Paracetamol
d. Adding sugar, honey or jam to feeding bottles
e. Moisturiser to the chin for skin irritation due to drooling

Answer: $\mathrm{d}$. Adding sugar, honey or jam to a feeding bottle or dipping a dummy in these substances has not been shown to be beneficial and can lead to tooth decay. Teething rings and rubbing the gums can provide temporary pain relief due to application of pressure to irritated areas; however, teething rings should be used with caution and parents should always follow manufacturers' instructions. Sugar-free paracetamol solution is the systemic medication of choice for pain or mild fever but should be used only in recommended dosages. Irritation of skin secondary to drooling can be a cause of significant discomfort in infants.

2. Salicylic acid works by inhibiting the activity of which enzyme?
a. Cyclooxygenase
b. Dihydrofolate reductase
c. Kinase
d. Methyltransferase
e. Phosphodiesterase 
Answer: a. Salicylic acid has anti-inflammatory effects as a result of irreversible inactivation of cyclooxygenase (COX), an enzyme responsible for production of prostaglandins and other proinflammatory markers. The remaining options are enzymes affected by various drugs, but not salicylic acid.

3. Which of the following abnormalities is usually NOT associated with salicylate toxicity?

a. Impaired platelet function

b. Elevated international normalised ratio (INR)

c. Hypocalcaemia

d. Hypokalaemia

e. Hypoglycaemia

Answer: c. Salicylates block formation of thromboxane A2, impairing activation of new platelets and platelet aggregation. Salicylate toxicity can inhibit formation of vitamin K-dependent clotting factors. Respiratory alkalosis is the primary acid-base disturbance in the early stages of acute toxicity. With increased loss of bicarbonate ions, the kidneys attempt to conserve hydrogen ions in preference to potassium ions, leading to hypokalaemia. Serum glucose levels can vary with salicylate toxicity. Hyperglycaemia can result from catecholamine-induced glycogenolysis and gluconeogenesis, and hypoglycaemia can result as glucose stores are depleted. Hypocalcaemia is not typically seen in salicylate toxicity.

\section{References}

1. Hoffman RS, Howland MA, Lewin NA, Nelson LS, Goldfrank LR. Goldfrank's toxicologic emergencies. 10th ed. McGraw-Hill Education, New York, 2015.

2. Reye RD, Morgan G, Baral J. Encephalopathy and fatty degeneration of the viscera. A disease entity in childhood. Lancet. 1963; 2: 749-52.

3. National Notifiable Diseases Surveillance System (NNDSS). Reye syndrome_1990 case definition. USA: Centers for Disease Control and Prevention, 2015. Available from: https://wwwn.cdc.gov/nndss/conditions/reye-syndrome/case-definition/1990 [accessed 3 Aug 2017]. 
4. Powell HC, Rosenberg RN, McKellar B. Reye's syndrome: isolation of parainfluenza virus. Report of three cases. Arch. Neurol. 1973; 29: 135-9.

5. Oman TK, Stewart MC, Burns A, Lang TF. Topical choline salicylates implicated in Reye syndrome. BMJ. 2008; 336: 1376.

6. GOV.UK. Oral salicylate gels: not for use in those younger than age 16 years. United Kingdom: Medicines and Health products Regulation Agency, 2009. Available from: https://www.gov.uk/drug-safety-update/oral-salicylate-gels-not-for-use-in-those-younger-thanage-16-years [accessed 3 Aug 2017].

7. Tein I. Impact of fatty acid oxidation disorders in child neurology: from Reye syndrome to Pandora's box. Dev. Med. Child Neurol. 2015; 57: 304-6.

8. McIntyre GT, McIntyre GM. Teething troubles? Br. Dent. J. 2002; 192: 251-5.

9. Bonjela. Bonjela Teething Gel. Australia: Reckitt Benckiser, 2017. Available from: http://www.bonjela.com.au/bonjela-teething-gel.php [accessed 3 Aug 2017].

10. Pearlman BL, Gambhir R. Salicylate intoxication: a clinical review. Postgrad. Med. 2009; 121: $162-8$

11. Williams GD, Kirk EP, Wilson CJ, Meadows CA, Chan BS. Salicylate intoxication from teething gel in infancy. MJA. 2011; 194: 146-8. 


\section{University Library}

\section{- M M N E R VA A gateway to Melbourne's research publications}

Minerva Access is the Institutional Repository of The University of Melbourne

Author/s:

Trung, N;Cranswick, N;Rosenbaum, J;Gelbart, B;Tosif, S

Title:

Chronic use of teething gel causing salicylate toxicity

Date:

2018-05-01

Citation:

Trung, N., Cranswick, N., Rosenbaum, J., Gelbart, B. \& Tosif, S. (2018). Chronic use of teething gel causing salicylate toxicity. JOURNAL OF PAEDIATRICS AND CHILD HEALTH, 54 (5), pp.576-578. https://doi.org/10.1111/jpc.13861.

Persistent Link:

http://hdl.handle.net/11343/283697 